\title{
BMJ Open Acute stress of the healthcare workforce during the COVID-19 pandemic evolution: a cross-sectional study in Spain
}

\author{
José Joaquín Mira (D) , ${ }^{1,2}$ Irene Carrillo (D) , ${ }^{1,3}$ Mercedes Guilabert (D) , ${ }^{1}$ \\ Aurora Mula, ${ }^{3}$ Jimmy Martin-Delgado (D) , ${ }^{3}$ Maria Virtudes Pérez-Jover (i) , 1 \\ Maria Asunción Vicente (D) , ${ }^{4}$ César Fernández (D) , ${ }^{4}$ on behalf of the SARS-CoV-2 \\ Second Victim Study Group
}

To cite: Mira JJ, Carrillo I, Guilabert M, et al. Acute stress of the healthcare workforce during the COVID-19 pandemic evolution: a cross-sectional study in Spain. BMJ Open 2020;10:e042555. doi:10.1136/ bmjopen-2020-042555

- Prepublication history and additional material for this paper are available online. To view these files, please visit the journal online (http://dx.doi org/10.1136/bmjopen-2020042555).

Received 08 July 2020 Revised 10 0ctober 2020 Accepted 15 October 2020

\section{Check for updates}

(c) Author(s) (or their employer(s)) 2020. Re-use permitted under CC BY-NC. No commercial re-use. See rights and permissions. Published by BMJ.

${ }^{1}$ Health Psychology, Miguel Hernandez University of Elche, Elche, Valenciana, Spain ${ }^{2}$ Alicante-Sant Joan Health District, Alicante, Spain

${ }^{3}$ Foundation for the Promotion of Health and Biomedical Research of Valencia Region (FISABI0),

San Juan, Spain

${ }^{4}$ Department of Systems Engineering and Automation, Miguel Hernandez University of Elche, Elche, Valenciana, Spain

Correspondence to Dr José Joaquín Mira; jose.mira@umh.es

\section{ABSTRACT}

Objectives To determine the volume of health professionals who suffered distress due to their care of patients with COVID-19 and to analyse the direction in which the response capacity of the professionals to face future waves of COVID-19 is evolving.

Design A cross-sectional study.

Setting Primary care and hospitals in Spain.

Participants A non-randomised sample of 685 professionals (physicians, nurses and other health staff).

Primary and secondary outcome measures Frequency and intensity of stress responses measured by the Acute Stress of Health Professionals Caring COVID-19 Scale (EASE). Variation of stress responses according to the number of deaths per day per territory and the evolutionary stage of the COVID-19 outbreak measured by the Kruskal-Wallis and the Mann-Whitney U tests.

Results The average score on the EASE Scale was 11.1 (SD 6.7) out of 30. Among the participants, $44.2 \%$ presented a good emotional adjustment, $27.4 \%$ a tolerable level of distress, $23.9 \%$ medium-high emotional load and $4.5 \%$ extreme acute stress. The stress responses were more intense in the most affected territories (12.1 vs 9.3 , $\mathrm{p}=0.003$ ) and during the disillusionment phase (12.7 vs 8.5 impact, 10.2 heroic and 9.8 honeymoon, $p=0.000$ ). Conclusions The pandemic has affected the mental health of a significant proportion of health professionals which may reduce their resilience in the face of future waves of COVID-19. The institutional approaches to support the psychological needs of health professionals are essential to ensure optimal care considering these results.

\section{INTRODUCTION}

As of 26th of August, COVID-19 pandemic has caused 819830 deaths worldwide, 28924 in Spain. ${ }^{1}$ The number of professionals suffering from COVID-19 is substantial. In Spain, it accounts for $21 \%$ of the total number of people infected. ${ }^{2}$

Although the incidence of the pandemic has expanded differently, among the

\section{Strengths and limitations of this study}

- This is an observational study to determine the volume of health professionals who present a high level of acute stress due to their care of patients with COVID-19 that may prevent them from carrying out their functions, and to analyse the direction in which the response capacity of the professionals to face future waves of COVID-19 is evolving.

- This study used a scale specifically designed to assess acute stress of health professionals in direct contact with patients with COVID-19 (EASE Scale). This scale was previously validated.

- The study was conducted in Spain between 18 March and 17 May 2020, coinciding with the phase of greatest acceleration and subsequent flattening of the curve of the pandemic. In this study, it has been shown how the impact of the first outbreak has left the workforce emotionally drained, which could limit their ability to adequately play their role in the face of a possible outbreak.

- The scale was not administered to a random sample of the population, which could limit the generalisability of the results. Also, the scale may have reached different sectors of the study population unevenly due to the means used to distribute it.

- Only basic sociodemographic data were collected from health professionals. No comparisons among subgroups were calculated.

geographical areas of each country, most hospitals and healthcare centres around the world have had to reorganise themselves to prioritise the care of patients with COVID-19, breaking with their usual work dynamics. In addition to this cause of work-related stress, there has been uncertainty in decisionmaking and a lack of resources to adequately treat patients and protect against possible contagion. ${ }^{3}{ }^{4}$ These circumstances have 
posed an additional risk to patient safety, ${ }^{5}$ which may have adversely affected quality of healthcare. ${ }^{6}$

The intensity of compassion fatigue, ${ }^{7}$ post-traumatic stress $^{89}$ and moral injury ${ }^{10} 11$ observed among professionals can be expected to depend on the intensity of the spread of the pandemic, the resources available and individual differences in stress response. Likewise, the extent of trauma experienced by professionals may also be influenced by factors that are not directly related to the healthcare response, such as family income and living situation, self-perceived health status, gender, personality traits and coping styles. ${ }^{12} 13$

Results of studies quantifying the magnitude of the impact of care of patients with COVID-19 on the mental health of healthcare professionals have been published since the beginning of the pandemic. These findings have varied widely due to the heterogeneity of the methodologies and instruments used. ${ }^{14}$

In the first studies, carried out at the beginning of February, $71.5 \%$ of healthcare personnel, mostly from the province of Hubai in China, presented emotional discomfort, ${ }^{15}$ with frequent depressive symptoms (55.7\%), anxiety responses $(44.7 \%)^{16}$ and insomnia (78.4\%). ${ }^{17}$ In Italy, in the days before the peak of infections (end of March), 49.4\% of health professionals reported symptoms of post-traumatic stress. ${ }^{18}$ In Ecuador, in the second half of April, $90 \%$ of the medical and nursing staff already presented moderate-severe burnout levels. ${ }^{19}$ In Spain, after the first wave of hospital care (April-May), 79.5\% and $51.1 \%$ of health professionals presented symptoms of anxiety and depression, respectively. ${ }^{20}$ The expansion of the pandemic in each territory has determined the magnitude of the emotional response. In China, sleep disorders and psychological symptoms were more frequent among medical staff in Wuhan than among staff in Ningbo. ${ }^{21} \mathrm{~A}$ recent meta-analysis showed that depression, anxiety and psychological distress were common responses in health professionals during the COVID-19 outbreak, is more likely in women and in those who had direct contact with positive cases of COVID-19. ${ }^{22}$

The magnitude and exceptionality of the situation justify these results. The experience of the crisis affects the entire staff and all professional levels, including support staff in healthcare (information technology, suppliers, janitors and so on). The complete absence of impact in mental health on the staff of health institutions would be difficult to explain. However, the most important question is not the number of professionals who have been emotionally affected as a result of their assistance services, a circumstance that has been aggravated by this crisis but is inherent to the work they do, but rather how many have not managed to recover, how their resilience is evolving or to what extent they can deal with a possible new outbreak.

Most studies have analysed the emotional responses in a short period (approximately 1 week) coinciding with a specific stage of the crisis. However, studies on community coping with catastrophic situations have described that the psychological response evolves resulting in: impact phase, heroic (intensification of efforts), honeymoon (optimism), disillusionment (fatigue) and restoration (recovery pre-crisis levels) ${ }^{23}$ Therefore, it is expected that the effects of the pandemic on the psychological response of health professionals will vary as the pandemic evolves and affect their resilience to a new outbreak. At the moment, there are no known studies that have addressed the problem from this perspective.

The objectives of this study were, first, to determine the volume of healthcare professionals who, because of the impact of the COVID-19 pandemic on the healthcare environment in which they work, experienced an excessive level of acute stress that prevented them from performing their role. Second, to analyse the direction in which the levels of the emotional response of professionals evolve to face a new outbreak, considering the variation in the frequency and intensity of their stress reactions in the different phases of the pandemic and according to the areas with the greater or lesser impact of the pandemic.

\section{METHODS}

A cross-sectional observational study in a nonrandomised sample of Spanish healthcare professionals was conducted. The study was designed to analyse two assumptions. First, since the results yielded in studies conducted elsewhere involving healthcare workforce caring for patients with COVID-19, it was expected that between $3 \%$ and $10.5 \%^{91324}$ of the healthcare professionals present psychological distress, with it being more severe as the pandemic becomes more intense. So, as seen in other studies, in those territories most affected by the pandemic, the percentage of professionals with emotional distress is expected to be higher. ${ }^{21}$ Finally, since the impact of the pandemic should be directly related to the distress experienced by professionals, it was expected that there will be a cumulative effect whereby the percentage of professionals with high levels of stress will be greater in the more advanced phases of the model of the psychological response during a disaster. ${ }^{23}$

The study was conducted in Spain between 18 March and 17 May 2020, coinciding with the phase of greatest acceleration and subsequent flattening of the curve of the pandemic.

\section{Variables and instrument}

We used a scale specifically designed to assess acute stress of healthcare professionals in direct contact with patients with COVID-19 (EASE Scale) (online supplemental material). This scale was previously validated, first, a pragmatic literature review of items assessing acute stress in healthcare professionals was conducted for possible inclusion, also, the most relevant sources of acute stress, pointed by the professional's experiences were represented into 17 reactive items; this number was finally reduced to 10 items, once participants considered their representativeness and comprehension. The instrument was validated following COSMIN protocol involving 228 Spanish 
physicians and nurses, it is composed of 10 items to which responses are given using a 4-level Likert-type scale $(0=\mathrm{It}$ is not happening to me, $1=\mathrm{It}$ happens to me in concrete situations, $2=$ It often happens to me and $3=\mathrm{I}$ am like this all the time). The total score on the scale can range from 0 to 30 points, with greater scores being interpreted as higher levels of stress. Reliability was calculated using OMEGA (0.87) and Cronbach's alpha (0.85). The items were grouped by Exploratory Factor Analysis into two factors that evaluate: affective response and fears and anxiety, explaining $55 \%$ of the variance. Factor 1, referring to the affective response, is composed of 6 items, so that the direct score on this factor ranges from 0 to 18 points. The factor 2 that evaluates fears and anxiety is composed of 4 items and its minimum and maximum possible scores are 0 and 12, respectively. The interpretability of the score ranges was established: $0-9$ points (good emotional adjustment), 10-14 points (emotional distress), 15-24 points (medium-high emotional overload), $>25$ points (extreme acute stress). ${ }^{25}$

\section{Participants}

Healthcare professionals from primary care centres and hospitals. At the time the study was conducted, the entire public health system was involved in the care of patients with COVID-19. Care for patients suffering other pathologies was suspended except for emergencies and those that could not be delayed, in other situations care was provided by telephone. We determined a minimum sample size of 650 professionals, considering a population of 392667 health professionals (hospitals and primary care), ${ }^{26}$ an effect size of 0.20 , a statistical power of $95 \%$ and a confidence level of $95 \%$.

\section{Patient and public involvement}

Patients or the public were not involved in any phase of this study.

\section{Procedure}

The diffusion of the scale and data gathering was done in a twofold way. First, the scale was made accessible through a web-based resource repository created by the authors to reduce the impact of the SARS-CoV-2 pandemic on the psychological well-being of healthcare professionals. ${ }^{27}$ These resources to cope with acute stress during the worst moments of the pandemic were disseminated through several Spanish scientific societies, social media and specialised press news. Second, the scale was accessible through the mobile application Be+ against COVID 2829 which was disseminated using the same means and by leaders of occupational health and hospital patient safety units. The consenting procedure to participate in the study was inherent in the use of the website and app.

\section{Acute stress responses}

Scores on the scale equal to or higher than 15 points were considered the level of stress with the potential to limit the professional's optimal performance of his/her function or work activity.

\section{Pandemic extension and acute stress responses}

The results of the self-assessment using this scale were linked to the data on the evolution of the pandemic in Spain using the data published daily by the Spanish Health Ministry, considering both the differences in impact between territories and the temporal phases of its evolution.

To determine the territories most and least affected by the pandemic on 17th of May 2020, the country was divided into two groups according to the number of deaths from COVID19. The first group included Madrid and Catalonia, with more than 5000 deaths. The second group included Asturias, the Balearic Islands, the Canary Islands, Cantabria, Extremadura, La Rioja, Murcia and Navarre with less than 500 deaths. To compare results on the EASE Scale according to the territory, a subsample of 336 participants working in health institutions in the regions specified above was selected. The comparison was made between the most and least affected territories.

\section{Acute stress during the outbreak evolution}

To analyse acute stress during the pandemic, four moments of the evolution of the outbreak were determined according to the number of deaths per day: less than 500 (18 March-25 March), between 600 and 900 (28 March-15 April), between 300 and 600 (16 April-26 April) and less than 300 (27 April-17 May). The periods described corresponded to the phases of the community's psychological response to the pandemic: impact (awareness of the problem, less than 500 deaths/day), heroic (increased efforts to cope with the crisis and mitigate the impact, between 600 and 900 deaths/day), honeymoon (hope, between 300 and 600 deaths/day) and disillusionment (accumulated fatigue, less than 300 deaths/ day). For the temporal definition of the phases, data on deaths per day were extracted from the dashboard of the Spanish Health Ministry.

\section{Statistical analysis}

Descriptive and frequency analyses were performed. Mean scores on each factor were transformed to a $0-10$ scale to allow comparison because the number of items was different on each factor. The Kruskal-Wallis test and the Mann-Whitney $\mathrm{U}$ test were used to determine the differences in acute stress reactions according to the time of evolution of the pandemic and the degree to which the territory was affected, respectively. Also, responses on the Be+ against COVID app and the website were compared. The comparative analyses of scores on the EASE Scale were conducted item by item, by scale factors and overall score. The Confidence Interval (CI) used was $95 \%$. Data coding and analysis was performed using IBM SPSS Statistics software, V.25.

\section{RESULTS}

A total of 685 professionals responded. Of these, 28.6\% $(\mathrm{n}=196)$ were doctors, $39 \%(\mathrm{n}=267)$ were nurses and $32.3 \%$ $(\mathrm{n}=222)$ were other healthcare staff (including advanced technicians in nursing auxiliary care, radiodiagnosis and clinical diagnostic laboratory). A majority of them reported working in a hospital setting $81.9 \%(\mathrm{n}=561)$, in primary care 
Table 1 EASE scores on the COVID-19 Acute Stress in Care Scale

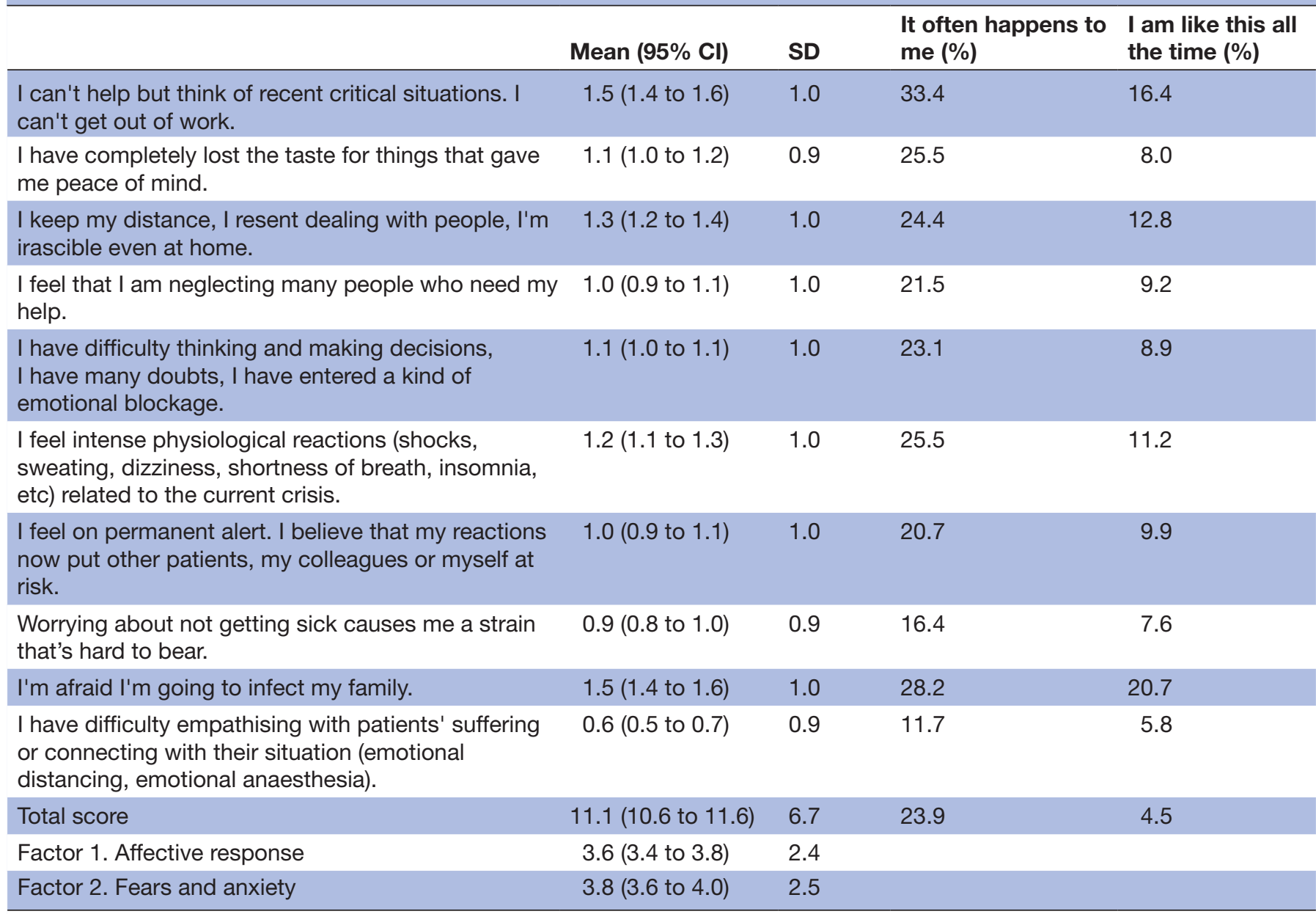

$\mathrm{N}=685$.

Scores from 0 to 3 points on each of the items on the scale.

Scores from 0 to 30 in total on the scale.

Score transformed from 0 to 10 points in factor 1

Score transformed from 0 to 10 points in factor 2 .

Mean difference between factors $p=0.2$.

$8 \%(\mathrm{n}=55)$ and in both care levels $10.1 \%(\mathrm{n}=69)$. A total of $40.4 \%$ worked in areas where the pandemic had had a greater impact. Most of them worked in Madrid (37\%), Valencia $(15.7 \%)$, Andalusia $(14.1 \%)$ and Catalonia $(3.3 \%)$.

\section{Scores on the EASE Scale}

The mean total score on the scale was 11.1 points (SD 6.7, 95\% CI 10.6 to 11.6 , range $0-30$ ), with $23.9 \%$ ( $\mathrm{n}=164$ ) with a medium-high level of emotional load, and $4.5 \% \quad(n=31)$ showing an extreme level of acute stress. Scores between the emotional response factor versus the fear/anxiety factor no differences were observed, 3.6 ( $\mathrm{SD} 2.4)$ versus $3.8(\mathrm{SD}=2.5)$; $\mathrm{p}=0.2$ (score transformed into a scale of $0-10$ points).

Three hundred and forty-one (49.8\%) of the health professionals highlighted that they had difficulties in being able to disconnect from work and 49\% ( $\mathrm{n}=335)$ expressed fear of infecting their family once they returned home at the end of the working day. Twenty-three per cent $(n=157)$ expressed concerns about not falling ill and 17\% ( $\mathrm{n}=116)$ experienced difficulties in empathising with the suffering of the patients (table 1).

Scores on the EASE Scale were similar among the professional categories $(\mathrm{p}=0.46)$. Only, differences were found between the scores for the statement related to maintaining emotional distance with people $(\mathrm{p}=0.03)$. Nurses scored higher than doctors or others.

Differences were found in the scores, in the use of the two platforms website versus Be+ against COVID app to respond to the EASE Scale (10.5; SD 6.3 vs 11.8; SD 7.1; $\mathrm{p}=0.008$ ). However, the use of the app was mostly employed by professionals from territories with greater expansion of the pandemic $66 \%(\mathrm{n}=206)$ versus $21.1 \%(\mathrm{n}=79)$. A total of $45.5 \%(\mathrm{n}=312)$ answered the questionnaire through the app and $54.5 \%(\mathrm{n}=373)$ through the website.

EASE Scale scores in territories with a higher incidence rate The average score on the EASE Scale was higher (up to $30 \%$ more) in those territories with a higher number of 
Table 2 Mean difference on EASE Scale between territories most and least affected by the SARS-CoV-2 pandemic

\begin{tabular}{|c|c|c|c|}
\hline & $\begin{array}{l}\text { Most affected } \\
\text { territories }\end{array}$ & $\begin{array}{l}\text { Least affected } \\
\text { territories† }\end{array}$ & $P$ value \\
\hline I can't help but think of recent critical situations. I can't get out of work. & 1.6 & 1.4 & 0.06 \\
\hline I keep my distance, I resent dealing with people, I'm irascible even at home. & 1.4 & 1.1 & 0.004 \\
\hline I feel that I am neglecting many people who need my help. & 1.1 & 0.9 & 0.12 \\
\hline $\begin{array}{l}\text { I have difficulty thinking and making decisions, I have many doubts, I have entered a kind of } \\
\text { emotional blockage. }\end{array}$ & 1.2 & 1.0 & 0.37 \\
\hline $\begin{array}{l}\text { I feel intense physiological reactions (shocks, sweating, dizziness, shortness of breath, } \\
\text { insomnia, etc) related to the current crisis. }\end{array}$ & 1.3 & 0.8 & 0.00 \\
\hline $\begin{array}{l}\text { I feel on permanent alert. I believe that my reactions now put other patients, my colleagues or } \\
\text { myself at risk. }\end{array}$ & 1.1 & 0.7 & 0.02 \\
\hline Worrying about not getting sick causes me a strain that's hard to bear. & 1.0 & 0.6 & 0.004 \\
\hline Factor 1. Affective response & 3.9 & 3.3 & 0.09 \\
\hline Factor 2. Fears and anxiety & 4.2 & 2.8 & 0.00 \\
\hline
\end{tabular}

$\mathrm{N}=336$.

Scores from 0 to 3 points on each of the items on the scale.

Scores from 0 to 30 in total on the scale.

Score transformed from 0 to 10 points in factor 1 .

Score transformed from 0 to 10 points in factor 2.

*Madrid and Catalonia (more than 5000 deaths by 17th of May 2020).

†Asturias, the Balearic Islands, the Canary Islands, Cantabria, Extremadura, La Rioja, Murcia and Navarre (less than 500 deaths by 17 th of May 2020).

recorded deaths compared with those territories that had a lower number (12.1 vs $9.3 \mathrm{p}=0.003$ ) (table 2). Despite the different affectation between territories, there were aspects in which these differences were not observed, such as completely losing the taste for things that previously produced tranquillity or well-being $(\mathrm{p}=0.50)$, feeling that people who required the help of the professional were being neglected $(\mathrm{p}=0.37)$, feeling emotionally blocked $(\mathrm{p}=0.37)$ or having difficulties in empathising with the patients' suffering ( $\mathrm{p}=0.93)$.

\section{EASE Scale scores according to the evolution of the pandemic and the different phases of psychological response to the disaster}

The average scores on the EASE Scale were higher in the disillusionment phase (27 April-17 May 2020) compared with the first period defined as the impact or awareness phase (18 March-25 March 2020) (12.7 vs $8.5 \mathrm{p}<0.0001$ ) (table 3).

\section{DISCUSSION}

Acute stress was manifested mainly by the inability to disconnect from work and the fear of infecting loved ones. Losing empathy for the suffering of patients and fear of becoming ill are the statements that probably best discriminate against professionals whose condition prevents them from continuing with their care work. This study backs up what has been suggested in previous studies that approximately $5 \%$ of the healthcare professionals suffered an extreme level of acute stress as a consequence of caring for patients with COVID-19. ${ }^{9} 1324$ Considering these results, the targets for the interventions designed to cope with distress due to the COVID-19 pandemic ${ }^{30}$ must take in account that approximately a quarter of professionals could need support not to evolve towards situations of extreme acute stress.

\section{Intense emotional responses in territories with a higher incidence rate}

This research suggests the level of acute stress experienced by Spanish professionals is higher as the damage from COVID-19 increases in patients. As expected, acute stress has been higher in those territories where the pandemic has had a greater impact in terms of the incidence of COVID-19 cases and deaths from this disease.

Those professionals working in territories where the pandemic has been particularly aggressive show more intense emotional responses in those elements related to thoughts, fears and physiological reactions because of the situation they are living. This result has not been observed with the fact of being emotionally blocked to think and take decisions or with the difficulty to empathise with the suffering of patients, these emotional responses could be developed in later stages. ${ }^{31}$ 
Table 3 Mean difference at four temporal moments of expansion of the SARS-COV-2 pandemic

\begin{tabular}{|c|c|c|c|c|c|}
\hline & Impact* & Heroict & Honeymoon¥ & Disillusionment§ & $P$ value \\
\hline $\begin{array}{l}\text { I can't help but think of recent critical situations. I } \\
\text { can't get out of work. }\end{array}$ & 1.3 & 1.4 & 1.3 & 1.6 & $<0.001$ \\
\hline $\begin{array}{l}\text { I keep my distance, I resent dealing with people, I'm } \\
\text { irascible even at home. }\end{array}$ & 0.5 & 1.1 & 1.1 & 1.5 & $<0.001$ \\
\hline $\begin{array}{l}\text { I have difficulty thinking and making decisions, } \\
\text { I have many doubts, I have entered a kind of } \\
\text { emotional blockage. }\end{array}$ & 0.8 & 0.9 & 0.9 & 1.3 & $<0.001$ \\
\hline $\begin{array}{l}\text { I feel intense physiological reactions (shocks, } \\
\text { sweating, dizziness, shortness of breath, insomnia, } \\
\text { etc) related to the current crisis. }\end{array}$ & 0.7 & 1.0 & 1.1 & 1.4 & $<0.001$ \\
\hline $\begin{array}{l}\text { Worrying about not getting sick causes me a strain } \\
\text { that's hard to bear. }\end{array}$ & 0.9 & 0.9 & 0.8 & 1.0 & 0.22 \\
\hline I'm afraid I'm going to infect my family. & 1.6 & 1.3 & 1.3 & 1.7 & $<0.001$ \\
\hline $\begin{array}{l}\text { I have difficulty empathising with patients' suffering } \\
\text { or connecting with their situation (emotional } \\
\text { distancing, emotional anaesthesia). }\end{array}$ & 0.4 & 0.7 & 0.5 & 0.6 & 0.42 \\
\hline Total score & 8.5 & 10.2 & 9.8 & 12.7 & $<0.001$ \\
\hline Factor 1. Affective response & 2.4 & 3.3 & 3.3 & 4.1 & $<0.001$ \\
\hline
\end{tabular}

$\mathrm{N}=685$.

Scores from 0 to 3 points on each of the items on the scale.

Scores from 0 to 30 in total on the scale.

Score transformed from 0 to 10 points in factor of 1 .

Score transformed from 0 to 10 points in factor 2 .

${ }^{*}$ From 18th of March to 25th of March 2020 (less than 500 deaths per day).

†From 28th of March to 15th of April 2020 (between 600 and 900 deaths per day).

\#From 16th of April to 26th of April 2020 (between 300 and 600 deaths per day).

$\S$ From 27th of April to 17th of May 2020 (less than 300 deaths per day).

Distress, therefore, appears to be associated with the pressures and demands caused by the pandemic, although it has not been possible to determine whether increased availability of resources or support programmes might have alleviated their effects.

\section{Intense emotional responses in the final phases of psychological response according to the evolution of the pandemic}

The evolution observed in the stress response of professionals is largely in line with the phases proposed by the psychological disaster response model. ${ }^{23}$ The level of acute stress manifested by professionals in the disillusionment phase is greater than the stress experienced during the impact phase. This result confirms the expected outcome and is suggesting that the capacity to deal with a new outbreak will be diminished if there is not enough time between outbreaks to allow for recovery or if decisive action is not taken to recover.

\section{Purpose-built measure}

This study used a scale specifically designed to discriminate between situations that cause acute stress in the course of caring for patients with COVID-19, unlike other studies that used scales to screen for symptoms of anxiety and depression. ${ }^{15233}$ This scale was based on the premise that the response to the consequences of the pandemic could not leave professionals indifferent and that the sources of stress that could disable professional duties would be quite different from those included in most instruments designed for other purposes. This differential element must be considered when interpreting the results, given that most of the studies that have so far evaluated the psychological impact of the COVID-19 
pandemic on healthcare professionals have used questionnaires that were validated under different conditions than the current ones. The EASE Scale has been sensitive to these changes, allowing the impact of the pandemic on healthcare professionals to be assessed ${ }^{10} 11$ and it can be expected to be useful for measuring the effects on emotional response and coping capacity if there is a resurgence.

This scale has reflected, above all, that they were unable to disconnect from work, experienced irritability, anxiety, fear of infecting their families and doubts about their ability to make decisions in clinical practice. However, most of the scores reported by healthcare professionals were in the first and second range of the scale (mild level of emotional distress). These data show that most professionals have not experienced, according to the EASE Scale scores, levels of extreme acute stress. This result suggests that we must differentiate between the emotional impact that can be expected from the stress of the crisis and that other emotional impact that prevents the responsibilities of the profession from being carried out with the appropriate guarantees for patients. These results confirm the existence of emotional discomfort in the staff, identifies in what this discomfort translates to, and that only 1 out of 20 professionals have been emotionally overwhelmed and with difficulties in carrying out their work.

In the case of a new outbreak, the data suggest that to determine the level of impact on the mental health of healthcare professionals, the following should be considered: employing instruments used to identify the sources of stress or to measure acute stress associated with the care of patients with COVID-19 rather than instruments designed for screening anxiety or depression; measurements should consider the care pressure faced by professionals and the evolution of this pressure over time because that is when it decreases when the intensity of acute stress increases.

\section{Applications of this study}

The SARS-CoV-2 pandemic has caused an unprecedented health crisis that has shaken the foundations of health systems around the world, requiring responses that were not always prepared. One reflection is the number of professionals infected. In Spain, as of 18 June, 52036 health professionals had contracted the COVID-19 disease and just over 13\% of those hospitalised required admission to the intensive care unit. ${ }^{2}$ This fact, added to the emotional response to the health crisis, has led to their being identified as the second victim of SARS-CoV-2.

The term 'second victim' ${ }^{34}$ applied to healthcare personnel has been used over the last two decades to refer to the emotional distress experienced by healthcare professionals when they suspect that they have been involved in a safety incident that has resulted in harm to the patient or when they observe that the patient in their care is not developing properly and their decisions and actions are being questioned. In the current scenario, where the healthcare professional has not had the appropriate means to cure and care for patients, we extend the concept of the second victim to refer to any healthcare or support professional involved in the care of people affected by COVID-19, who presents acute stress responses when continuously exposed to an extreme situation caused by the combination of a series of critical factors, including social alarm, oversaturation of services, scarcity of resources and the poor evolution of the patients under their care.

The response to the emotional and psychological needs that the staff of healthcare institutions is experiencing as a result of this situation is justified not only on ethical grounds but also to ensure quality care and patient safety. ${ }^{35}$ Precisely the recovery of these systems after the COVID-19 crisis that requires restoring the working morale and welfare of health professionals and strengthening their capacity for resilience. ${ }^{36}$ Some authors suggest adopting measures based on the social support provided by coworkers or peers. ${ }^{3738}$ Digital initiatives have also been developed in the form of broader programmes that integrate social support as one of their resources to mitigate the impact of COVID-19 on healthcare professionals. ${ }^{1030}$

Despite the recent emergence of tools to measure the effects of the pandemic on mental health and behaviour in the general population, ${ }^{39-41}$ there are still no specific measures designed and validated for evaluation in healthcare professionals. As far as we are aware, this study is the first to explore the emotional distress caused by the COVID-19 health crisis and one of the first to use a specifically validated measure for this purpose.

\section{Limitations}

This study was conducted using a scale that was not administered to a random sample of the population which could limit the generalisability of the findings. During the pandemic, depending on the care needs of the territories, primary care professionals moved to work in hospitals (eg, field hospitals). The scale may have reached different sectors of the study population unevenly due to the media used. Access to the scale by participants via their well-being repository may have over-represented the response of professionals who were feeling more distressed. Those days the entire health system was dedicated to the care of patients with COVID-19. No specific procedure was used to confirm that respondents to the scale were working caring for patients with COVID-19 at the time of the outbreak, despite prior instructions requiring this. The motivation of respondents and those who chose not to respond could have biased the sample and therefore the results. The study looked at a limited number of sociodemographic variables with the intention that participants would feel that their privacy was guaranteed when completing the scale. This decision was made because, during the first wave of COVID-19 in Spain, most healthcare professionals were reluctant to receive help in managing their acute stress. Consequently, the collection of some sociodemographic data (such as gender or age) could have been a barrier for them to self-assess their 
stress levels due to the fear of being identified. At that time, to give emotional support to our healthcare workforce was prioritised. These precautions in data collection significantly limited the possibilities of national and international comparative analyses of stress responses by groups (such as sex, age, experience and so on). It also prevented intrasubject comparisons at different times of the crisis. Despite these limitations, the results obtained are in line with those found in other studies. ${ }^{9} 1324$ The comparative analyses between the most and least affected territories only took into account the number of deaths/ day without controlling for other variables that could be influencing the impact of the pandemic on the healthcare centre and its professionals, such as access to equipment, human resources, among others. It should be considered that during the pandemic, there was an increase in personnel and resources throughout the health system in response to an emergency that could not be quantified. The training of this staff to perform their new function could not be considered which could affect their stress levels.

\section{CONCLUSION}

Over time, we have become more scientifically and technically prepared to deal with COVID-19 and have learnt multiple lessons on how to best deal with this crisis, but the impact of the first outbreak has left the workforce emotionally drained, which could limit their ability to properly perform their role in the face of a possible outbreak. Consequently, healthcare institutions in the process of workforce recovery must incorporate measures to restore the well-being and work morale of healthcare professionals. This study demonstrates this, confirming that emotional difficulties begin to appear at the end of the most critical phases of the pandemic.

Twitter José Joaquín Mira @notasalpie, Irene Carrillo @icarrillo_umh, Mercedes Guilabert @MerceGuilabert, Aurora Mula @AuroraMula, Jimmy Martin-Delgado @ jimmymartin2, Maria Virtudes Pérez-Jover @vipejo2012, Maria Asunción Vicente @ suniumh and César Fernández @cesarfperis

Collaborators SARS-CoV-2 Second Victim Study Group: Ángel Cobos-Vargas, Olga Beatriz Martínez-García (Hospital Universitario Clínico San Cecilio de Granada, Andalucía, Spain), Ma José Bueno-Domínguez (Grup SAGESSA, Reus, Spain), María Pilar Astier-Peña (Centro de Salud 'La Jota', Zaragoza, Spain), Pastora Pérez-Pérez (Sociedad Española de Calidad Asistencial, Spain), Matilde Lahera-Martín, Carmen Silvestre-Busto (Servicio Navarro de Salud-Osasunbidea), Susana Lorenzo-Martínez (Hospital Universitario Fundación Alcorcón, Madrid, Spain), Ascensión SánchezMartínez (Hospital General Universitario Reina Sofía, Murcia, Spain), Bárbara MarcoGómez, Cristina Abad-Bouzán (Zona de Salud de Calatayud, Zaragoza, Spain), Carlos Aibar-Remón (Hospital Clínico Universitario Lozano Blesa, Zaragoza), and Jesús María Aranaz-Andrés (Hospital Universitario Ramón y Cajal).

Contributors JJM conceived and designed the work. JJM, IC, MG and MVP-J designed the scale. MAV and CF developed the web and mobile app for the online administration of the scale. JM was responsible for the recruitment of participants. AM performed the statistical analysis. IC and MG wrote the first draft of the manuscript, which was critically reviewed for important intellectual content by JJM and JM. All authors reviewed the draft and approved the final version of the manuscript.

Funding The authors have not declared a specific grant for this research from any funding agency in the public, commercial or not-for-profit sectors.
Competing interests None declared.

Patient consent for publication Not required.

Ethics approval The study protocol was approved by the Research Committee of the San Juan University Hospital in Alicante (8th of April 2020).

Provenance and peer review Not commissioned; externally peer reviewed.

Data availability statement Data are available upon reasonable request.

Supplemental material This content has been supplied by the author(s). It has not been vetted by BMJ Publishing Group Limited (BMJ) and may not have been peer-reviewed. Any opinions or recommendations discussed are solely those of the author(s) and are not endorsed by BMJ. BMJ disclaims all liability and responsibility arising from any reliance placed on the content. Where the content includes any translated material, BMJ does not warrant the accuracy and reliability of the translations (including but not limited to local regulations, clinical guidelines, terminology, drug names and drug dosages), and is not responsible for any error and/or omissions arising from translation and adaptation or otherwise.

Open access This is an open access article distributed in accordance with the Creative Commons Attribution Non Commercial (CC BY-NC 4.0) license, which permits others to distribute, remix, adapt, build upon this work non-commercially, and license their derivative works on different terms, provided the original work is properly cited, appropriate credit is given, any changes made indicated, and the use is non-commercial. See: http://creativecommons.org/licenses/by-nc/4.0/.

\section{ORCID iDs}

José Joaquín Mira http://orcid.org/0000-0001-6497-083X

Irene Carrillo http://orcid.org/0000-0002-6981-7284

Mercedes Guilabert http://orcid.org/0000-0002-0706-9911

Jimmy Martin-Delgado http://orcid.org/0000-0003-4384-9197

Maria Virtudes Pérez-Jover http://orcid.org/0000-0001-9089-0497

Maria Asunción Vicente http://orcid.org/0000-0002-8630-7251

César Fernández http://orcid.org/0000-0002-9391-9192

\section{REFERENCES}

1 Johns Hopkins University \& Medicine. Coronavirus resource center. COVID-19 Dashboard by the center for systems science and engineering (CSSE) at Johns Hopkins University (JHU). Available: https://coronavirus.jhu.edu/map.html [Accessed 08 Jul 2020].

2 Corporación Radio y Televisión Española (rtve). Los profesionales sanitarios contagiados de COVID-19 superan Los 52.000, 81 en La última semana. Available: https://www.rtve.es/noticias/20200619/ profesionales-sanitarios-contagiados-covid-19-superan-50000/ 2014047.shtml [Accessed 19 Jun 2020].

3 Binkley CE, Kemp DS. Ethical rationing of personal protective equipment to minimize moral residue during the COVID-19 pandemic. J Am Coll Surg 2020;230:1111-3.

4 Steinberg E, Balakrishna A, Habboushe J, et al. Calculated decisions: COVID-19 calculators during extreme resource-limited situations. Emerg Med Pract 2020;22:CD1-5.

5 Hall LH, Johnson J, Watt I, et al. Healthcare staff wellbeing, burnout, and patient safety: a systematic review. PLoS One 2016;11:e0159015.

6 Wallace JE, Lemaire JB, Ghali WA. Physician wellness: a missing quality indicator. Lancet 2009;374:21.

7 Alharbi J, Jackson D, Usher K. The potential for COVID-19 to contribute to compassion fatigue in critical care nurses. J Clin Nurs 2020;29:2762-4.

8 Restauri N, Sheridan AD. Burnout and posttraumatic stress disorder in the coronavirus disease 2019 (COVID-19) pandemic: intersection, impact, and interventions. J Am Coll Radiol 2020;17:921-6.

9 Huang JZ, Han MF, Luo TD, et al. [Mental health survey of medical staff in a tertiary infectious disease hospital for COVID-19]. Zhonghua Lao Dong Wei Sheng Zhi Ye Bing Za Zhi 2020;38:192-5.

10 Williamson V, Murphy D, Greenberg N. COVID-19 and experiences of moral injury in front-line key workers. Occup Med 2020;70:kqaa052.

11 Williams RD, Brundage JA, Williams EB. Moral injury in times of COVID-19. Health Serv Psychol 2020:1-5.

12 DiGangi JA, Gomez D, Mendoza L, et al. Pretrauma risk factors for posttraumatic stress disorder: a systematic review of the literature. Clin Psychol Rev 2013;33:728-44.

13 Guo J, Wu P, Tian D, et al. Post-Traumatic stress disorder among adult survivors of the Wenchuan earthquake in China: a repeated cross-sectional study. J Anxiety Disord 2014;28:75-82. 
14 Pappa S, Ntella V, Giannakas T, et al. Prevalence of depression, anxiety, and insomnia among healthcare workers during the COVID-19 pandemic: a systematic review and meta-analysis. Brain Behav Immun 2020;88:S0889-1591(20)30845-X

15 Lai J, Ma S, Wang Y, et al. Factors associated with mental health outcomes among health care workers exposed to coronavirus disease 2019. JAMA Netw Open 2020;3:e203976.

16 Zhang C, Yang L, Liu S, et al. Survey of insomnia and related social psychological factors among medical staff involved in the 2019 novel coronavirus disease outbreak. Front Psychiatry 2020;11:306.

17 Qi J, Xu J, Li B-Z, et al. The evaluation of sleep disturbances for Chinese frontline medical workers under the outbreak of COVID-19. Sleep Med 2020;72:1-4.

18 Rossi R, Socci V, Pacitti F, et al. Mental health outcomes among frontline and second-line health care workers during the coronavirus disease 2019 (COVID-19) pandemic in Italy. JAMA Netw Open 2020;3:e2010185.

19 Vinueza Veloz AF, Aldaz Pachacama NR, Mera Segovia CM, et al. Síndrome de burnout en médicos/as $Y$ enfermeros/as ecuatorianos durante La pandemia de COVID-19, 2020. Available: https://www. google.com/url?sa=t\&rct=j\&q=\&esrc=s\&source=web\&cd=\&ved= 2ahUKEwiH8bCK5ZXqAhXTiFwKHfTxDmMQFjAAegQIAhAB\&url= https\%3A\%2F\%2Fpreprints.scielo.org\%2Findex.php\%2Fscielo\% 2Fpreprint\%2Fdownload\%2F708\%2F958\%2F988\&usg=AOvVaw3P SHAVyRBM1rCJu6IL_xWI

20 Universidad Autónoma de Madrid. El 79,5\% de los sanitarios sufren ansiedad y el $40 \%$ se siente emocionalmente agotado tras la primera oleada de atención hospitalaria por Covid-19 [press statement]. Available: https://www.ucm.es/file/estres-sanitarios-mayo-2020

$21 \mathrm{Li} \mathrm{X}, \mathrm{Yu} \mathrm{H}$, Bian G, et al. Prevalence, risk factors, and clinical correlates of insomnia in volunteer and at home medical staff during the COVID-19. Brain Behav Immun 2020;87:140-1.

22 Chou R, Dana T, Buckley DI, et al. Epidemiology of and risk factors for coronavirus infection in health care workers: a living rapid review. Ann Intern Med 2020;173:120-36.

23 Myers D, Zunin L. Phases of disaster, in DeWolfe D (ED.).Training manual for mental health and human service workers in major disasters. Washington, DC: US Government Printing Office, 2000.

24 Lu W, Wang H, Lin Y, et al. Psychological status of medical workforce during the COVID-19 pandemic: a cross-sectional study. Psychiatry Res 2020;288:112936.

25 Mira JJ, Cobos-Vargas A, Martínez-García O, et al. The acute stress scale in healthcare professionals caring for patients with COVID-19. validation study. Health Economics \& Outcomes Research 2020.

26 Ministerio de Sanidad, Consumo y Bienestar Social. ortal Estadístico, Área de Inteligencia de Gestión. Consulta Interactiva del Sistema Nacional de Salud [Internet], 2018. Available: https://pestadistico.inte ligenciadegestion.mscbs.es/publicoSNS/Comun/DefaultPublico.aspx
27 SARS-CoV-2 Second Victim Study Group. Be+ against COVID. SARS-CoV-2 (COVID-19) second victims. Available: https:// secondvictimscovid19.umh.es/p/resource-09.html

28 BE+ against COVID. Google Play [Internet, mobile app]. Available: https://play.google.com/store/apps/details?id=com.appandabout. defusing

29 BE+ against COVID. App Store [Internet, mobile app]. Available: https://apps.apple.com/es/app/ser-positivo-contra-covid/ id1512478131

30 Blake H, Bermingham F, Johnson G, et al. Mitigating the psychological impact of COVID-19 on healthcare workers: a digital learning package. Int J Environ Res Public Health 2020;17:2997.

31 Kadhum M, Farrell S, Hussain R, et al. Mental wellbeing and burnout in surgical trainees: implications for the post-COVID-19 era. $\mathrm{Br} J$ Surg 2020;107:e264.

32 Liu C-Y, Yang Y-Z, Zhang X-M, et al. The prevalence and influencing factors in anxiety in medical workers fighting COVID-19 in China: a cross-sectional survey. Epidemiol Infect 2020;148:e98.

$33 \mathrm{Wu} \mathrm{Y,} \mathrm{Wang} \mathrm{J,} \mathrm{Luo} \mathrm{C,} \mathrm{et} \mathrm{al.} \mathrm{A} \mathrm{comparison} \mathrm{of} \mathrm{burnout} \mathrm{frequency}$ among oncology physicians and nurses working on the frontline and usual wards during the COVID-19 epidemic in Wuhan, China. J Pain Symptom Manage 2020;60:e60-5.

34 Wu AW. Medical error: the second victim. The doctor who makes the mistake needs help too. BMJ 2000;320:726-7.

35 Grant S, Davidson J, Manges K, et al. Creating healthful work environments to deliver on the quadruple AIM. J Nurs Adm 2020;50:314-21.

36 Rangachari P, L. Woods J, Resilience PO. Preserving organizational resilience, patient safety, and staff retention during COVID-19 requires a holistic consideration of the psychological safety of healthcare workers. Int J Environ Res Public Health 2020;17:E1267.

37 Wu AW, Connors C, Everly GS. COVID-19: peer support and crisis communication strategies to promote institutional resilience. Ann Intern Med 2020;172:M20-1236.

38 Albott CS, Wozniak JR, McGlinch BP, et al. Battle Buddies: rapid deployment of a psychological resilience intervention for health care workers during the COVID-19 pandemic. Anesth Analg 2020;131:43-54

39 Ahorsu DK, Lin CY, Imani V, et al. The fear of COVID-19 scale: development and initial validation. Int $J$ Ment Health Addict 2020:1-9.

40 Chandu V, Pachava S, Vadapalli V, et al. Development and initial validation of the COVID-19 anxiety scale. Indian J Public Health 2020;64:201-4.

41 Taylor S, Landry CA, Paluszek MM, et al. Development and initial validation of the COVID stress scales. J Anxiety Disord 2020;72:102232. 